\title{
Dolor en niños y adolescentes con enfermedades neuromusculares
}

\author{
M. López y J. Miró \\ Unidad para el Estudio del Dolor-ALGOS. Centre de Recerca en Avaluació i Mesura de la Conducta. \\ Departamento de Psicología. Insitut d'Investigació Sanitària Pere Virgili. Universitat Rovira i Virgili. \\ Reus (Tarragona)
}

López M, Miró J. Dolor en niños y adolescentes con enfermedades neuromusculares. Rev Soc Esp Dolor 2013; 20(3): 142-149.

\begin{abstract}
The aim of this paper is to review the available information on the experience of pain in children and adolescents with neuromuscular disease (NMD). In order to do this, we examined the MEDLINE and SCOPUS databases and located published articles on the subject until March 2012. After a thorough analysis, we identified 33 articles that met the inclusion criteria. The analysis conducted shows that pain is a very common experience for these young people, usually moderate to severe, with a high frequency of occurrence and long lasting. In general, these are chronic conditions. These chronic pain problems affect the quality of life of young people, beyond what could be explained by the NMD alone. The reviewed studies show that pain not only negatively impacts the quality of life of young people, also that of their caregivers is at jeopardy.
\end{abstract}

Key words: Pain: Neuromuscular disease. Children. Adolescents. Systematic review.

Financiación-Agradecimientos: Este trabajo ha sido posible gracias, en parte, a las ayudas del Ministerio de Ciencia e Innovación (PSI2009 12193PSIC), la Fundació La Marató de TV3, RecerCaixa, y de la Agència de Gestió d'Universitats i de Recerca, Generalitat de Catalunya (2009 SGR 434).

Recibido: 17-09-12.

Aceptado: 01-12-12.

\section{RESUMEN}

El objetivo de este trabajo es revisar la información disponible sobre la experiencia de dolor en niños y adolescentes con una enfermedad neuromuscular (ENM). Para ello, se examinaron las bases de datos SCOPUS y MEDLINE y se localizaron los artículos publicados sobre el tema hasta marzo de 2012. Tras un análisis exhaustivo, se identificaron 33 artículos que cumplían con los criterios de inclusión. Del análisis efectuado se desprende que el dolor es una experiencia muy habitual en estos jóvenes, que se trata de molestias, por lo general, de moderadas a severas, con una alta frecuencia de aparición y de larga duración. Habitualmente se trata de cuadros crónicos. Estos problemas afectan a la calidad de vida de los jóvenes, más allá de lo que puede explicar la propia ENM. Los estudios revisados muestran que no solo la calidad de vida de los jóvenes se ve afectada por la presencia del dolor, también la de sus cuidadores.

Palabras claves: Dolor. Enfermedades neuromusculares. Niños. Adolescentes. Revisión sistemática.

\section{INTRODUCCIÓN}

Las enfermedades neuromusculares (ENM) son un grupo de enfermedades neurológicas hereditarias o adquiridas cuya principal característica es la pérdida progresiva de fuerza muscular y la degeneración del conjunto de los músculos y de los nervios que los controlan. Se pueden presentar en cualquier etapa de la vida, forman parte del grupo de enfermedades llamadas raras, pues afectan a un pequeño porcentaje de la población (1).Las ENM comprenden un conjunto de más de 150 enfermedades neurológicas que se caracterizan por su gran diversidad. En España se calcula que existen más de 40.000 afectados, la prevalencia aproximada es de unas 100 personas por cada 100.000 habitantes (2). Aunque las molestias que experimentan estas personas varían en función del tipo de ENM, 
en general, presentan problemas de movilidad, de sueño, y en otros aspectos inherentes al bienestar, como las relaciones sociales o el desempeño académico $(3,4)$. Igualmente, informan de problemas de funcionamiento psicológico y de graves interferencias en todas las áreas de la vida $(5,6)$. Aunque escasas, las investigaciones disponibles muestran que el dolor es un problema frecuente e importante en las personas con una ENM $(7,8)$. En general, la información sobre lo que sucede en población pediátrica es menor que la relacionada con los adultos. Sin embargo, en los últimos años se han publicado una serie de estudios que permiten identificar al dolor, particularmente el dolor crónico, como un grave problema en esta población (9-17).El objetivo de este trabajo es revisar el estado de la cuestión sobre el estudio de dolor en niños y adolescentes con ENM, valorar los avances e identificar áreas para la investigación futura.

\section{MATERIAL Y MÉTODOS}

\section{Procedimiento}

Se realizó una búsqueda en las bases de datos Scopus y Medline desde su comienzo hasta marzo de 2012. Se utilizaron las palabras clave: "niños", "adolescentes", "dolor", "dolor pediátrico", "enfermedades neuromusculares", que se combinaron con otras palabras clave adicionales, específicamente: "calidad de vida", "sueño", "movilidad", "actividad", "apoyo social", "familia", "escuela", "fatiga", "evaluación”.

Las múltiples combinaciones de las palabras clave resultaron en 2.686 artículos. Tras esta primera criba, se comprobaba si los artículos en verdad presentaban alguna relación con el estudio del dolor en enfermedades neuromusculares en general, o con alguna ENM de forma más específica (por ejemplo, distrofia muscular congénita), y se verificaba la edad de la muestra estudiada, para confirmar que los estudios en cuestión considerasen a niños y/o adolescentes.

\section{Criterios de inclusión y exclusión}

Para incluir un artículo en esta revisión, este debía cumplir los siguientes criterios:

1. Versar sobre el dolor en enfermedades neuromusculares, independientemente de su naturaleza u objetivo.

2. Contar con una muestra formada por niños y/o adolescentes (los participantes en los estudios debían tener hasta 18 años de edad).

3. Estar escrito en español o inglés.

Los criterios de exclusión eran los siguientes:

1. Incluir diferentes grupos de pacientes, y ser imposible distinguir los datos relativos al grupo de jóvenes con ENM.

2. Tener una muestra formada también por adultos y no permitir identificar los valores relativos a los más jóvenes.

\section{RESULTADOS}

Finalmente, tras la valoración completa de los primeros hallazgos se identificaron 33 artículos que cumplían los criterios de inclusión; todos son trabajos que han aparecido en los últimos 10 años. El número total de sujetos que participaron en estos estudios fue de 2.845 (el mínimo de participantes en cualquier muestra empleada fue de 8 sujetos y el máximo de 1.296) (Tabla I), siendo el rango de edad de los grupos de participantes de 0 a 20 años. El $61 \%$ de los participantes, en los estudios en los cuales se informaba de este detalle, eran de sexo masculino.

Los resultados de esta revisión, y la información de las conclusiones de los trabajos analizados se organiza en dos bloques: (1) características epidemiológicas del dolor y (2) impacto del dolor en la calidad de vida de las personas con una ENM.

TABLA I. RESUMEN DE LOS ARTÍCULOS SOBRE DOLOR EN JÓVENES CON ENM

\begin{tabular}{lll}
\hline Autores & Tipo de ENM & Características de la muestra \\
\hline Arnaud y cols. (35)* & Parálisis cerebral & $\mathrm{N}=818$ \\
& & Edad $=8-12$ años \\
\hline Baiarnidi y cols. (28)** & Distrofia muscular de Duchenne & $\mathrm{N}=27$ \\
& & Masculino $=27$ \\
& & Edad $=$ media de 11,26 \\
\hline Berrin y cols. (14)* & Parálisis cerebral & $\mathrm{N}=73$ \\
& & Edad $=5$ a 18 años \\
\hline Bray y cols. (10) & Distrofia muscular de Duchenne & $\mathrm{N}=34$ \\
& & Masculino $=34$ \\
& & Edad $=10-18$ años
\end{tabular}


TABLA I (CONT.). RESUMEN DE LOS ARTÍCULOS SOBRE DOLOR EN JÓVENES CON ENM

\begin{tabular}{|c|c|c|}
\hline Autores & Tipo de ENM & Características de la muestra \\
\hline Butbul y cols. $(9)^{* *}$ & Dermatomiositis & $\begin{array}{l}\mathrm{N}=33 \\
\text { Masculino }=13 \\
\text { Femenino }=20 \\
\text { Edad }=12,6 \text { años }\end{array}$ \\
\hline Davis y cols. (29) & Parálisis cerebral & $\begin{array}{l}\mathrm{N}=17 \\
\text { Masculino }=9 \\
\text { Femenino }=8 \\
\text { Edad }=13 \text { a } 18 \text { años }\end{array}$ \\
\hline Engel y cols. (25) & Distrofia muscular de Duchenne & Revisión \\
\hline Engel y cols. (26) & Parálisis cerebral & $\begin{array}{l}\mathrm{N}=20 \\
\text { Masculino }=11 \\
\text { Femenino }=9 \\
\text { Edad }=6 \text { a } 17 \text { años }\end{array}$ \\
\hline Engel y cols. (15) & $\begin{array}{l}\text { Distrofia muscular de Duchenne, distrofia muscular } \\
\text { miotónica, distrofia muscular de Becker, distrofia } \\
\text { muscular Limb-Girdle, distrofia muscular congénita, } \\
\text { Distrofia facioescápulo humeral, otras ENM }\end{array}$ & $\begin{array}{l}\mathrm{N}=42 \\
\text { Masculino }=24 \\
\text { Femenino }=18 \\
\text { Edad }=2-20 \text { años }\end{array}$ \\
\hline Hadden y Baeyer (17) & Parálisis cerebral & $\begin{array}{l}\mathrm{N}=43 \\
\text { Masculino }=22 \\
\text { Femenino }=21 \\
\text { Edad }=1 \text { a } 19 \text { años }\end{array}$ \\
\hline Hemmingsson y cols. (37)* & Distrofia muscular (no especifica) & $\begin{array}{l}\mathrm{N}=27 \\
\mathrm{Edad}=10 \text { a } 15 \text { años }\end{array}$ \\
\hline Higashimoto y cols. (47)* & Síndrome del dolor complejo regional & $\begin{array}{l}\mathrm{N}=8 \\
\text { Edad }=0-19 \text { años }\end{array}$ \\
\hline Houlihan y cols. (16) & Parálisis cerebral & $\begin{array}{l}\mathrm{N}=198 \\
\text { Masculino = } 115 \\
\text { Femenino }=83 \\
\text { Edad }=5 \text { a } 18 \text { años }\end{array}$ \\
\hline Liptak y cols. (33) & Parálisis cerebral & $\begin{array}{l}\mathrm{N}=235 \\
\text { Masculino }=137 \\
\text { Femenino }=98 \\
\text { Edad }=2 \text { a } 18 \text { años }\end{array}$ \\
\hline Mah y cols. (31) & $\begin{array}{l}\text { Distrofia muscular de Duchenne, distrofia muscular de } \\
\text { Becker, distrofia muscular congénita, distrofia muscular } \\
\text { miotónica, Distrofia facioescápulo humeral, miopatía } \\
\text { congénita, Charcot- Marie-Tooth, miopatía metabólica, } \\
\text { otras miopatías y otras distrofias musculares }\end{array}$ & $\begin{array}{l}\mathrm{N}=109 \\
\text { Masculino }=75 \\
\text { Femenino }=34 \\
\text { Edad }=2-18 \text { años }\end{array}$ \\
\hline Parkes y cols. (34) & Parálisis cerebral & $\begin{array}{l}\mathrm{N}=818 \\
\text { Masculino }=471 \\
\text { Femenino }=328 \\
\text { Edad }=7 \text { a } 12 \text { años }\end{array}$ \\
\hline Parkes y cols. (22) & Parálisis cerebral & $\begin{array}{l}\mathrm{N}=785 \\
\text { Masculino }=463 \\
\text { Femenino }=322 \\
\text { Edad }=8 \text { a } 12 \text { años }\end{array}$ \\
\hline Parkinson y cols. (12)* & Parálisis cerebral & $\begin{array}{l}\mathrm{N}=1.296 \\
\mathrm{Edad}=8 \text { a } 12 \text { años }\end{array}$ \\
\hline
\end{tabular}


TABLA I (CONT.). RESUMEN DE LOS ARTÍCULOS SOBRE DOLOR EN JÓVENES CON ENM

\begin{tabular}{|c|c|c|}
\hline Autores & Tipo de ENM & Características de la mиеstra \\
\hline Ramstad y cols. (11) & Parálisis cerebral & $\begin{array}{l}\mathrm{N}=153 \\
\text { Masculino }=81 \\
\text { Femenino }=72 \\
\text { Edad }=8 \text { a } 18 \text { años }\end{array}$ \\
\hline Riquelme y Montoya (20) & Parálisis cerebral & $\begin{array}{l}\mathrm{N}=15 \\
\text { Masculino }=8 \\
\text { Femenino }=7 \\
\text { Edad }=5 \text { a } 14 \text { años }\end{array}$ \\
\hline Riquelme y cols. $(21)^{*}$ & Parálisis cerebral & $\begin{array}{l}\mathrm{N}=51 \\
\text { Edad }=6 \text { a } 17 \text { años }\end{array}$ \\
\hline Straub y cols. (48) & Distrofia muscular Limb-Girdle & Revisión \\
\hline Svedberg y cols. (18) & Parálisis cerebral & $\begin{array}{l}\mathrm{N}=107 \\
\text { Masculino }=60 \\
\text { Femenino }=47 \\
\text { Edad }=5 \text { a } 13 \text { años }\end{array}$ \\
\hline Takaso y cols. (38)* & Distrofias musculares congénitas & $\begin{array}{l}\mathrm{N}=10 \\
\text { Edad }=11-17 \text { años }\end{array}$ \\
\hline Takaso y cols. (39)* & Distrofia muscular de Duchenne & $\begin{array}{l}\mathrm{N}=20 \\
\text { Edad }=11-17 \text { años }\end{array}$ \\
\hline Tsirikos y Baker (49) & Atrofia espinal muscular & Revisión \\
\hline Tsirikos (19) & Miopatías & Revisión \\
\hline Vuillerot y cols. (24) & $\begin{array}{l}\text { Distrofia muscular de Duchenne, amiotrofia muscular } \\
\text { espinal, miopatía congénita, distrofia muscular } \\
\text { congénita, distrofia muscular Limb-Girdle, distrofia } \\
\text { facioescápulo humeral, neuropatía periférica }\end{array}$ & $\begin{array}{l}\mathrm{N}=43 \\
\text { Masculino }=32 \\
\text { Femenino }=11 \\
\text { Edad }=10-17 \text { años }\end{array}$ \\
\hline Wagner y cols. (23) & $\begin{array}{l}\text { Amiotrofia muscular espinal, distrofia muscular } \\
\text { Duchenne, enfermedad de Pompe, distrofia muscular } \\
\text { congénita }\end{array}$ & $\begin{array}{l}\mathrm{N}=12 \\
\text { Masculino }=9 \\
\text { Femenino }=3 \\
\text { Edad }=\text { no especifica }\end{array}$ \\
\hline White-Koning y cols. (30) & Parálisis cerebral & $\begin{array}{l}\mathrm{N}=500 \\
\text { Masculino }=286 \\
\text { Femenino }=214 \\
\text { Edad }=8 \text { a } 12 \text { años }\end{array}$ \\
\hline White-Koning y cols. (32) & Parálisis cerebral & $\begin{array}{l}\mathrm{N}=204 \\
\text { Masculino }=127 \\
\text { Femenino }=77 \\
\text { Edad }=8 \text { a } 12 \text { años }\end{array}$ \\
\hline Young y cols. (36) & Parálisis cerebral & $\begin{array}{l}\mathrm{N}=28 \\
\text { Masculino }=15 \\
\text { Femenino }=13 \\
\text { Edad }=8 \text { a } 13 \text { años }\end{array}$ \\
\hline Zebracki y Drotar, (13) & $\begin{array}{l}\text { Distrofia muscular de Duchenne, distrofia muscular de } \\
\text { Becker. }\end{array}$ & $\begin{array}{l}\mathrm{N}=53 \\
\text { Masculino }=53 \\
\text { Edad }=8-18 \text { años }\end{array}$ \\
\hline
\end{tabular}

*No realizan distinción con respecto al sexo. **No indican rango de edad 


\section{Características epidemiológicas}

Informar sobre la extensión del dolor en las ENM así, en general, resulta complicado, por la multitud de enfermedades que comprende esta rúbrica. No obstante, los estudios disponibles muestran que el dolor es un problema habitual e importante en las ENM. En general, e independientemente del problema estudiado, más de la mitad de las muestras de participantes informan experimentar dolor. Así, por ejemplo, en el caso del estudio de Zebracki \& Drotar (13) las cifras varían desde el $54 \%$ para la distrofia muscular de Duchenne (DMD) hasta el $80 \%$ para la distrofia muscular de Becker (DMB). Las características de la propia enfermedad contribuyen al impacto y la severidad del dolor. Así, por ejemplo, Svedberg y cols. (18) informan de diferencias significativas en la prevalencia de problemas de dolor en una muestra de niños con parálisis cerebral según su capacidad para deambular: el $48 \%$ de niños que podían andar informaban tener dolor, frente al $79 \%$ de aquellos que no podían hacerlo. Igualmente, estos autores constataron que el $94 \%$ de los niños con dolor tenían este problema hacía más de un año. De forma parecida, Tsirikos (19) informa de importantes diferencias en el dolor que experimentan personas con distrofia muscular con y sin deformidad espinal: 90 y $67 \%$ respectivamente. Los estudios en los que se han comparado grupos de pacientes con una ENM y sujetos sanos, se informa de un mayor porcentaje de personas con dolor entre aquellas con una ENM (p. ej., 20), así como de una mayor intensidad de dolor y sensibilidad al tacto (p. ej., 21). En la mayoría de estudios la intensidad del dolor informado suele ser moderada, aunque también se reflejan casos de dolor severo $(9,11,12,17,21-26)$. Algunos trabajos apuntan relaciones estadísticamente significativas entre intensidad del dolor y edad, esto es, a mayor edad, mayor intensidad del dolor (p. ej., 12). En general, la frecuencia de los episodios de dolor es elevada, en muchos casos prácticamente diaria (p. ej., 13, 15-18, 25, 26).

La duración, como la frecuencia, es muy variable. Los estudios identifican episodios desde 1 minuto hasta 2 días de duración (25). Sin embargo, la mayoría de los trabajos relatan episodios de dolor que duran varias horas (p. ej., 13, 15, 17).

A pesar de la diversidad de las ENM y que el dolor varía en su localización, el lugar más habitual es las piernas (25), si bien también son habituales las localizaciones siguientes: espalda, caderas o zona pélvica, pies, cuello, hombros, brazos, manos y cabeza $(11,13,15,25)$. El malestar aparece asociado a las zonas con mayor intensidad de dolor $(10,11,25)$.

\section{Calidad de vida}

La calidad de vida, según la Organización Mundial de la Salud (27), es el completo bienestar físico, men- tal y social, y no solo la ausencia de enfermedad. Se trata, por lo tanto, de un constructo multidimensional y subjetivo, donde la apreciación de la persona sobre su funcionamiento tiene un papel importante. Los estudios sobre los efectos del dolor en la calidad de vida de jóvenes con una ENM son escasos (4). No obstante, es igualmente cierto que la información disponible demuestra que el impacto negativo del dolor en la calidad de vida de estas personas es muy importante. Así, por ejemplo, los informes publicados recogen que los niveles de calidad de vida observados son menores que en la población general, especialmente en áreas concernientes al funcionamiento físico, así como a la participación en las tareas de la escuela y/o del hogar $(10,13-16,25,28$ 36). También se registra una tendencia a puntuaciones bajas en el área de la salud psicosocial, lo que sugiere que estos jóvenes presentan ciertas dificultades emocionales y sociales $(13,21,31,34)$.

Los estudios disponibles se han preocupado por los efectos del dolor en la calidad del sueño, en la movilidad de estas personas, así como en la calidad de vida de los cuidadores de los jóvenes afectados.

\section{Calidad del sueño}

En estos jóvenes, los problemas que les ocasionan la ENM (p. ej., las limitaciones para ajustarse a ciertas posiciones para dormir o la insuficiencia de las vías respiratorias) interfieren y dificultan unas pautas de sueño adecuadas (37). No obstante, el dolor se ha mostrado como un factor que interfiere significativamente en la calidad del sueño más allá de los problemas propios de la enfermedad $(25,26)$. Los estudios disponibles muestran, en efecto, que el dolor deteriora la calidad de sueño de estas personas, también la de sus cuidadores, y que contribuye a un círculo vicioso de dolor-fatiga-mala calidad de sueño-fatigadolor que resulta de difícil manejo $(9,18)$.

\section{Movilidad}

Entre las acciones/actividades que ocasionan dolor y provocan un mayor deterioro en la calidad de vida de estos jóvenes se encuentran las siguientes: andar/correr, sentarse, estar inmovilizado, estirarse, usar aparatos ortopédicos o férulas $(11,13,15,17,25,26)$. Independientemente de la ENM de referencia, el dolor interfiere en la actividad general, y es en el ámbito de la movilidad y en el autocuidado donde son más evidentes sus efectos perniciosos $(11,13,15,25,26)$. La asistencia a la escuela y el buen desempeño académico también se ven afectados por la presencia de dolor, más allá de lo que explicarían los efectos de la ENM $(9,14,16)$. Los procedimientos médicos 
y rehabilitadores para tratar la enfermedad (p. ej., terapia ocupacional, masajes, exámenes médicos, cirugía) son una fuente de dolor importante, y también contribuyen a estos efectos perniciosos (17).

\section{Calidad de vida de los cuidadores}

Los padres o cuidadores de los jóvenes con una ENM, también se suelen ver afectados por la enfermedad y las condiciones de sus hijos, tanto por el impacto que representa ver a un ser querido en unas condiciones difíciles como por el esfuerzo importante que en ocasiones representa tener cuidado de sus necesidades. Un impacto que aumenta a medida que progresa el deterioro de sus hijos (10). Por ejemplo, la atención parental nocturna es una atención habitual que deben prestar, especialmente en niños con distrofias musculares, y esto tiene una influencia directa en las pautas de sueño de los propios padres (15). De hecho, hay estudios que muestran que estas atenciones y cuidados nocturnos, más que incidir en el sueño de los niños, donde realmente impactan es en la calidad del sueño de los cuidadores, pues algunos de estos cuidados se realizan incluso cuando los niños están dormidos o semidormidos (37).

Las dificultades físicas y el deterioro en la deambulación (10) y el dolor (14), se encuentran entre las mayores preocupaciones de los padres. Y, así, son también factores importantes en el deterioro progresivo de su calidad de vida. Houlihan y cols. (16) informan de correlaciones positivas significativas entre la presencia de dolor en los hijos y el impacto emocional en los padres. Así mismo, Parkes y cols. (22) observan que los padres de niños con parálisis cerebral con dolor de moderado a severo tienen mayor probabilidad de presentar un alto nivel de estrés. En líneas similares, Parkinson y cols. (12) informan de relaciones significativas entre el desempleo parental y la severidad y frecuencia de los episodios de dolor de sus hijos. El estrés que experimentan los padres de niños con ENM parece estar relacionado, al menos en parte, con las responsabilidades que conlleva la atención de su hijo, pero también con la dificultad para atender las necesidades de los hijos, entre ellas, y de forma particular, el manejo del dolor.

\section{DISCUSIÓN}

La literatura disponible muestra que el dolor en los niños y adolescentes con una ENM es habitual, más aún, que el dolor es crónico. En efecto, más de la mitad de los participantes en los estudios publicados, e independientemente del tipo de ENM, informaban sentir dolor (entre el 54 y el $80 \%$, según los estudios) (13) y que la mayoría de estos se trataba de problemas de larga duración $(11,15,17,18,21,23,25,26,37-39)$. Buena parte de estas personas informa de un dolor de intensidad moderada en el mejor de los casos $(9,11-13,17,21-26)$, con una frecuencia de una a varias veces por semana $(13,15$ $18,25,26)$ y una duración media aproximada de varias horas $(13,15,17,25,26)$. La localización del dolor varía, aunque las piernas, la espalda, las caderas o región pélvica, pies, cuello, hombros y brazos son las más frecuentes $(11,13,15,25,26)$.

El dolor, aún siendo un problema en sí mismo, también lo es por el impacto que representa tanto para quien lo experimenta en primera persona, como para quien se encarga del cuidado de estos jóvenes. Fundamentalmente, el dolor interfiere en las actividades relacionadas con el funcionamiento físico o la movilidad (p. ej., andar, $11,13,15,17,25,26,31)$, el sueño $(11,13,15,25,26,37)$ y en el autocuidado (p. ej., vestirse, bañarse; 11,13,15,25,26). Igualmente, el dolor influye negativamente en el estado emocional de estas personas. Diferentes estudios han demostrado la capacidad predictiva de la intensidad del dolor respecto del funcionamiento emocional y de la autoestima $(9,13,28,31,34)$. Más aún, el dolor interfiere en el desarrollo integral de los jóvenes con una ENM, pues afecta negativamente a su capacidad para realizar actividades que son de vital importancia para un desarrollo social adecuado, como las actividades escolares, o el juego, sobre todo aquel que requiere de cierta actividad física $(9,13,21,31,34)$. Los trabajos publicados muestran una relación entre dolor y el funcionamiento escolar, específicamente con las faltas a la escuela, así como con un nivel bajo de desempeño académico $(14,16)$.

Los efectos del dolor se dejan notar muy pronto en la vida de estas personas. La probabilidad de que el dolor se extienda a otras zonas corporales una vez este aparece es alta $(4,15,40)$, como también lo es que el dolor se cronifique en estas particulares circunstancias. Una vez el dolor se cronifica es muy difícil eliminarlo. Así, pues, la atención y manejo del dolor debería ser tarea prioritaria en el tratamiento de estos pacientes, como también la prevención del dolor crónico (41).

Los efectos del dolor de los jóvenes con ENM trascienden sus límites individuales; el dolor acaba por afectar a todos aquellos con quienes conviven, particularmente a sus cuidadores. Así, se ha demostrado que los padres de estos niños y jóvenes pueden presentan problemas de sueño (37), ansiedad, estrés y otros trastornos emocionales $(10,12,16,22)$, incluso perder el empleo por las demandas que el cuidado de sus hijos les impone (12).

Si bien es verdad que el estudio del dolor en las ENM, y del impacto en la vida de estas personas, ha avanzado considerablemente en los últimos años, también lo es que las investigaciones con jóvenes (niños y adolescentes) todavía siguen siendo escasas. Se necesitan estudios epidemiológicos longitudinales que permitan no solo establecer correctamente la prevalencia del dolor, también los facto- 
res asociados con el progreso y mantenimiento del dolor y la discapacidad asociada.

En general, los estudios se centran en analizar la intensidad del dolor de los jóvenes con ENM $(9,10,12,21$ 24,30,34). Futuros trabajos también deberían analizar si otras dimensiones del dolor más allá de la intensidad (p. ej., localización, extensión, cualidad) están relacionados con la interferencia del dolor e incluso con el impacto psicosocial que provoca, como parece que sucede en los adultos (41).

El progreso en el estudio y tratamiento del dolor en los jóvenes con una ENM pasa, también, por identificar los mejores instrumentos para la evaluación, tanto del dolor y sus diferentes características, como de otras variables relacionadas (p. ej., estrategias de afrontamiento). Actualmente, los instrumentos que se utilizan no han sido contrastados para ser utilizados en estas poblaciones. Y si bien es plausible que sus propiedades psicométricas sigan siendo adecuadas en estas condiciones, no hay certeza alguna de que sea así. Por tanto, en el futuro sería deseable que se contrastaran las características de los instrumentos que se emplean en la evaluación de los jóvenes con ENM y dolor.

El tratamiento del dolor en las personas con una ENM, particularmente del dolor crónico, dista mucho de ser adecuado. Igual que ha sucedido en los últimos años en el ámbito de los adultos $(4,5,8,42-46)$, es de desear que con la población infanto-juvenil también se avance en el diseño y contraste de tratamientos adecuados. Dada la naturaleza multifactorial del dolor, estos deberían ser multidisciplinares.

Menospreciar el dolor y sus efectos en estas poblaciones ha sido moneda de uso corriente. En estos momentos, sin embargo, esta posición parece un sinsentido, dada la información que existe al respecto. El progreso en esta área no es nada fácil, investigar resulta complicado pues es difícil conseguir muestras amplias; se trata de enfermedades poco habituales. La colaboración de todos los interesados: pacientes, familiares, clínicos e investigadores es fundamental. En este sentido, mantener registros de pacientes resulta una estrategia de vital importancia.

\section{CORRESPONDENCIA: \\ Jordi Miró \\ Departamento de Psicología \\ Universitat Rovira i Virgili \\ Carretera de Valls, s/n \\ 43007 Tarragona \\ e-mail: jordi.miro@urv.cat}

\section{BIBLIOGRAFÍA}

1. Amato A, Russell J. Neuromuscular disorders. Estados Unidos: McGraw-Hill. 2008. p. 3-8.
2. Federación Española de enfermedades neuromusculares (ASEM). Guía de las enfermedades neuromusculares. Información y apoyo a las familias. Jaén: Ed. Formación Alcalá. 2008. p. 11-14.

3. Hornyak J, Pangilinan P. Rehabilitation of children and adults who have neuromuscular diseases. Phys Med Rehabil Clin N Am. 2007;18:883-97.

4. Carter G, Han J, Abresch R, et al. The importance of assesing quality of life in patients with neuromuscular disorders. Am J Hosp Palliat Care. 2007;23(6):493-7.

5. Miró J, Raichle KA, Carter GT, et al. Impact of biopsychosocial factors on chronic pain in in persons with myotonic and facioscapulohumeral muscular dystrophy. Am J Hosp Palliat Care. 2009;26:308-19.

6. Nieto R, Miró J, Huguet A, et al. Are coping and catastrophising independently related to disability and depression in patients with whiplash associated disorders? Disabil Rehabil. 2011;33(5):389-98.

7. Carter G, Miró J, Abresch R, et al. Disease burden in neuromuscular disease: the role of chronic pain. Phys Med Rehabil Clin N Am. 2012;23(3):719-29.

8. Jensen M, Hoffman A, Stoelb B, et al. Chronic pain in persons with myotonic dystrophy and facioscapulohumeral dystrophy. Arch Phys Med Rehabil. 2008;89:320-8.

9. Butbul Y, Stremler R, Benseler S, et al. Sleep, fatigue and the relationship to pain, disease activity and quality of life in juvenile idiopathic arthritis and juvenile dermatomyositis. Rheumatology. 2011;50:2051-60.

10. Bray P, Bundy A, Ryan M, et al. Health status of boys with Duchenne muscular dystrophy: A parent's perspective. J Paediatr Child Health. 2011;47:557-62.

11. Ramstad K, Jahnsen R, Skjeldal O, et al. Characteristics of recurrent musculoskeletal pain in children with cerebral palsy aged 8 to 18 years. Dev Med Child Neurol. 2011;53:1013-8.

12. Parkinson K, Gibson L, Dickinson H, et al. Pain in children with cerebral palsy: a cross-sectional multicentre European Study. Acta Paediatr. 2010;99:446-51.

13. Zebracki K, Drotar,D. Pain and activity limitations in children with Duchenne or Becker muscular dystrophy. Dev Med Child Neurol. 2008;50:546-52.

14. Berrin S, Malcarne V, Varni J, et al. Pain, fatigue and school functioning in children with cerebral palsy: a path-analytic model. J Pediatr Psychol. 2007;32(3):330-7.

15. Engel J, Kartin D, Jaffe K. Exploring chronic pain in youth with Duchenne muscular dystrophy: A model for pediatric neuromuscular disease. Phys Med Rehabil Clin N Am. 2005;16:1113-24.

16. Houlihan C, O'Donnell M, Conaway M, et al. Bodily pain and health-related quality of life in children with cerebral palsy. Dev Med Child Neurol. 2004;46:305-10.

17. Hadden K, Baeyer C. Pain in children with cerebral palsy: common triggers and expressive behaviors. Pain. 2002; 99:281-8.

18. Svedberg L, Englund E, Malker H, et al. Parental perception of cold extremities and other accompanying symptoms in children with cerebral palsy. Eur J Paediatr Neurol. 2008;12:89-96.

19. Tsirikos A. (iv) Development and treatment of spinal deformity in patients with neurological or myopathic conditions. J Orthop Trauma. 2011;25(6):425-34.

20. Riquelme I, Montoya P. Developmental changes in somatosensory processing in cerebral palsy and healthy individuals. Clin Neurophysiol. 2010;121:1314-20.

21. Riquelme I, Cifre I, Montoya P. Age-related changes of pain experiences in cerebral palsy and healthy individuals. Pain Med. 2011;12:535-45. 
22. Parkes J, Caravale B, Marcelli M, et al. Parenting stress and children with cerebral palsy: a European cross-sectional survey. Dev Med Child Neurol. 2011;53:815-21.

23. Wagner S, Poirot C, Vuillerot C, et al. Tolerance and effecttiveness on pain control of Pamidronate intravenous infusions in children with neuromuscular disorders. Ann Phys Rehabil Med. 2011;54:348-58.

24. Vuillerot C, Hodgkinson I, Bissery A, et al. Self-perception of quality of life by adolescents with neuromuscular diseases. J Adolesc Health. 2009;46:70-6.

25. Engel J, Kartin D, Carter G, et al. Pain in youths with neuromuscular disease. Am J Hosp Palliat Care. 2009;26(5):40512.

26. Engel J, Petrina T, Dudgeon B, et al. Cerebral palsy and chronic pain: a descriptive study of children and adolescents. Physical \& Occupational Therapy In Pediatrics. 2005; 25(4):73-84.

27. Organización Mundial de la salud (1948). Constitución de la organización mundial de la salud. [Documento en línea]. Disponible: http://apps.who.int/gb/bd/PDF/bd47/EN/constitution-en.pdf [Consulta: 2012, Mayo 27]

28. Baiardini I, Minetti C, Bonifacino S, et al. Quality of life in Duchenne muscular dystrophy: The subjective impact in children and parents. J Child Neurol. 2011;00:1-7.

29. Davis E, Shelly A, Waters E, et al. Quality of life of adolescents with cerebral palsy: perspectives of adolescents and parents. Dev Med Child Neurol. 2009;51:193-9.

30. White-Koning M, Arnaud C, Dickinson H, et al. Determinants of child-parent agreement in quality-of-life reports: a european study of children with cerebral palsy. Pediatrics. 2007; 120:e804.

31. Mah J, Thannhauser J, Kolski H, et al. Parental stress and quality of life in children with neuromuscular disease. Pediatr Neurol. 2007;39(2):102-7.

32. White-Koning M, Grandjean H, Colver A, et al. Parent and professional reports of the quality of life of children with cerebral palsy and associated intellectual impairment. Dev Med Child Neurol. 2008;50:618-24.

33. Liptak G, O'Donnell M, Conaway M, et al. Health status of children with moderate to severe cerebral palsy. Dev Med Child Neurol. 2001;43:364-70.

34. Parkes J, White-Koning M, Dickinson H, et al. Psychological problems in children with cerebral palsy: a cross-sectional European study. J Child Psychol Psychiatry. 2008; 49(4):405-13.

35. Arnaud C, White-Koning M, Michelsen S, et al. Parent-reported quality of life of children with cerebral palsy in Europe. Pediatrics. 2008;121;54-64.
36. Young B, Rice H, Dixon-Woods M, et al. A qualitative study of the health-related quality of life of disabled children. Dev Med Child Neurol. 2007;49:660-5.

37. Hemmingsson H, Stenhammar A, Paulsson K. Sleep problems and the need for parental night-time attention in children with physical disabilities. Child Care Health Dev. 2008;35(1):89-95.

38. Takaso M, Nakasawa T, Imura T, et al. Surgical correction of spinal deformity in patients with congenital muscular dystrophy. J Orthop Sci. 2010;15:493-501.

39. Takaso M, Nakasawa T, Imura T, et al. Two-years results for scoliosis secondary to Duchenne muscular dystrophy fused to lumbar 5 with segmental pedicle screw instrumentation. J Orthop Sci. 2010;15:171-7.

40. Strehle E. Long-term management of children with neuromuscular disorders. J Pediatr (Rio J). 2009;85(5).

41. Miró J, Gertz K, Carter G, et al. Chronic pain in neuromuscular disease: pain site and intensity differentially impacts function. Phys Med Rehabil Clin N Am. En prensa.

42. Nieto R, Raichle K, Jensen M, et al. Changes in pain-related psychosocial variables predict changes in outcomes in individuals with myotonic muscular dystrophy and facioscapulohumeral dystrophy. Clin J Pain. 2012;28:47-54.

43. Hosoi M, Molton I, Jensen M, et al. Relationships among alexithymia and pain intensity, pain interference, psychological functioning, and vitality in persons with neuromuscular disease: Considering the effect of negative affectivity. Pain. 2010;149:273-7.

44. Jensen M, Abresch R, Carter G, et al. Chronic pain in persons with neuromuscular disorders. Arch Phys Med Rehabil. 2005;86:1155-63.

45. Jensen M, Abresch R, Carter G. The reliability and validity of a self-reported version of the Functional Independence Measure in persons with neuromuscular disease and chronic pain. Arch Phys Med Rehabil. 2005;86:116-22.

46. Abresch R, Carter G, Jensen M, et al. Assessment of pain and health-related quality of life in slowly progressive neuromuscular disease. Am J Hosp Palliat Care. 2002;19:3948.

47. Higashimoto T, Baldwin E, Gold J, et al. Reflex sympathetic dystrophy: complex regional pain syndrome type I in children with mitocondrial disease and maternal inheritance. Arch Dis Child. 2008;93:390-7.

48. Straub V, Bushby K. The childhood limb-girdle muscular dystrophies. Semin Pediatr Neurol. 2006;13:104-14.

49. Tsirikos A, Baker A. Spinal muscular atrophy: Classification, aetiology, and treatment of spinal deformity in children and adolescents. Current Orthopaedics. 2006;20:430-45. 